\title{
Dynamic Crack Analysis in Isotropic/Orthotropic Media via Extended Isogeometric Analysis
}

\author{
Tiantang Yu, Yongling Lai, and Shuohui Yin \\ Department of Engineering Mechanics, Hohai University, 1 Xikang Road, Nanjing 210098, China \\ Correspondence should be addressed to Tiantang Yu; tiantangyu@hhu.edu.cn
}

Received 8 February 2014; Revised 13 March 2014; Accepted 13 March 2014; Published 8 April 2014

Academic Editor: Hung Nguyen-Xuan

Copyright (C) 2014 Tiantang Yu et al. This is an open access article distributed under the Creative Commons Attribution License, which permits unrestricted use, distribution, and reproduction in any medium, provided the original work is properly cited.

\begin{abstract}
The extended isogeometric analysis (X-IGA) is the combination of the extended finite element method (X-FEM) and the isogeometric analysis (IGA), so the X-IGA possesses the advantages of both methods. In this paper, the X-IGA is extended to investigate the dynamic stress intensity factors of cracked isotropic/orthotropic media under impact loading. For this purpose, a corresponding dynamic X-IGA model is developed, the Newmark time integration scheme is used to achieve a dynamic response, and the dynamic stress intensity factors are evaluated through the contour interaction integral technique. Numerical simulations show that the X-IGA results agree with other available reference solutions, and accurate results can be obtained by using the X-IGA with a relatively coarse mesh.
\end{abstract}

\section{Introduction}

Among existing numerical methods, the extended finite element method (X-FEM), which was developed in 1999 by Belytschko and his coworkers [1,2], is the most effective method for solving discontinuity problems. In the X-FEM, the geometry of the discontinuity is independent of the computational mesh; therefore, the computational mesh does not need to be updated in the simulation of the propagation of discontinuities. In the past decades, the extensive literature on improving and applying the original X-FEM in modeling discontinuities has been published [3-15]. The X-FEM aims to enrich standard finite element approximation by using discontinuous basis functions in the framework of partition of unity.

The standard finite element approximation is elementbased polynomial approximation, and it yields discretization errors in complex geometry. In order to overcome the drawback, the isogeometric analysis (IGA) was proposed by Hughes et al. [16] in 2005. The principle of the IGA is that nonuniform rational $\mathrm{B}$-splines (NURBS) basis functions are employed as shape functions for geometric description and field approximation. The IGA has some unique advantages [16], so it has been developed and applied in many fields including structural mechanics [17-24], solid mechanics [2527], fluid mechanics [28], and contact mechanics [29].

Recently, the IGA has been improved with enrichment functions to solve linear elastic fracture mechanics problems [30-32] and curved interface problems [33]. The extended isogeometric analysis (X-IGA) was proposed in [31] and contains the inherent advantages of both IGA and X-FEM. Currently, the X-IGA is further developed to analyze cracked orthotropic media [34]. In this study, the X-IGA is extended to investigate the dynamic fracture behavior of stationary cracks in isotropic/orthotropic media. A corresponding dynamic X-IGA model is developed, and the Newmark time integration scheme is used to achieve a dynamic response. The dynamic stress intensity factors (DSIFs) are evaluated by using the contour interaction integral technique.

The paper is organized as follows. Section 2 briefly reviews the fundamentals of NURBS-based IGA. The X-IGA for dynamic cracked isotropic/orthotropic media is described in Section 3. The DSIFs are derived by using the contour interaction integral technique in Section 4. Section 5 presents the numerical results obtained via X-IGA and compares the results with other solutions. Finally, conclusions and prospects are drawn in Section 6. 


\section{NURBS-Based Isogeometric Analysis}

In the CAD, a two-dimensional NURBS surface can be constructed as [35]

$$
\mathbf{S}(\xi, \eta)=\sum_{i=1}^{n} \sum_{j=1}^{m} R_{i, j}^{p, q}(\xi, \eta) \mathbf{B}_{i, j}
$$

where $R_{i, j}^{p, q}(\xi, \eta)$ is the NURBS basis function; $p$ and $q$ are, respectively, the orders of basis functions in the $\xi$ direction and the $\eta$ direction; $\mathbf{B}_{i, j}$ represents the coordinate of control point; and $n$ and $m$ are the numbers of basis functions in the $\xi$ direction and the $\eta$ direction, respectively.

In NURBS-based isogeometric analysis [16], the displacement field $\mathbf{u}$ is approximated similarly to the isoparametric finite element method as follows:

$$
\mathbf{u}=\sum_{I=1}^{n} R_{I}(\boldsymbol{\xi}) \mathbf{u}_{I}
$$

where $n$ is the number of the control points; $\xi$ is the parametric coordinate; $R_{I}(\xi)$ denotes the NURBS basis function at control point $I$; and $\mathbf{u}_{I}$ is the displacements of control point I.

\section{X-IGA for Dynamic Cracked Media}

3.1. Displacement Approximation. Similar to the X-FEM, the $\mathrm{X}$-IGA aims to enrich the standard IGA approximation by using additional functions on the basis of the partition of unity to model discontinuities. For crack problems, the enriched displacement approximation can be expressed as

$$
\begin{aligned}
\mathbf{u}^{h}(\mathbf{x})= & \sum_{i \in N^{s}} R_{i}(\mathbf{x}) \mathbf{u}_{i}+\sum_{j \in N^{\text {cut }}} R_{j}(\mathbf{x})\left[H(\mathbf{x})-H\left(\mathbf{x}_{j}\right)\right] \mathbf{a}_{j} \\
& +\sum_{k \in N^{\text {tip }}} R_{k}(\mathbf{x}) \sum_{\alpha=1}^{4}\left[F_{\alpha}(\mathbf{x})-F_{\alpha}\left(\mathbf{x}_{k}\right)\right] \mathbf{b}_{k \alpha},
\end{aligned}
$$

where $R_{i}(\mathbf{x}), R_{j}(\mathbf{x})$, and $R_{k}(\mathbf{x})$ are the shape functions of standard IGA (NURBS basis functions); $\mathbf{u}_{i}, \mathbf{a}_{j}$, and $\mathbf{b}_{k \alpha}$ are, respectively, the displacement and enrichment variable vectors at control point; $N^{s}, N^{\text {cut }}$, and $N^{\text {tip }}$ are, respectively, the set of all control points in the computational domain, the set of control points enriched with a modified Heaviside step function $H(\mathbf{x})$, and the set of control points enriched with the crack-tip branch enrichment functions $F_{\alpha}(\mathbf{x}) ;(\alpha=1, \ldots, 4) ;$ and the basis function support of the control point in $N^{\text {cut }}$ is completely split by the crack, whereas that of the control point in $N^{\text {tip }}$ is partly split by the crack.

The modified Heaviside step function $H(\mathbf{x})$ is given by

$$
H(\mathbf{x})= \begin{cases}+1 & \mathbf{x}>0 \\ -1 & \mathbf{x}<0\end{cases}
$$

The crack-tip branch enrichment functions $F_{\alpha}(\mathbf{x})$ for orthotropic materials are defined as [36]

$$
\left\{F_{\alpha}(\mathbf{x})\right\}_{\alpha=1}^{4}=\left\{\sqrt{r} \cos \frac{\theta_{1}}{2} \sqrt{g_{1}(\theta)} \quad \sqrt{r} \cos \frac{\theta_{2}}{2} \sqrt{g_{2}(\theta)} \sqrt{r} \sin \frac{\theta_{1}}{2} \sqrt{g_{1}(\theta)} \sqrt{r} \sin \frac{\theta_{2}}{2} \sqrt{g_{2}(\theta)}\right\},
$$

where $r$ and $\theta$ are the crack-tip local polar coordinates, and

$$
\begin{aligned}
g_{k}(\theta) & =\sqrt{\left(\cos \theta+\mu_{k x} \sin \theta\right)^{2}+\left(\mu_{k y} \sin \theta\right)^{2}}, \\
\theta_{k} & =\operatorname{arctg}\left(\frac{\mu_{k y} \sin \theta}{\cos \theta+\mu_{k x} \sin \theta}\right),
\end{aligned}
$$

where $\mu_{k x}$ and $\mu_{k y}(k=1,2)$ are crack-tip material parameters and $\mu_{k}=\mu_{k x}+i \mu_{k y}(k=1,2)$ are the roots of

$$
a_{11} \mu^{4}-2 a_{16} \mu^{3}+\left(2 a_{12}+a_{66}\right) \mu^{2}-2 a_{26} \mu+a_{22}=0
$$

where $a_{i j}$ is the compliance coefficients.

Equation (5) cannot be directly used for isotropic materials because of the presence of $0 / 0$ in the equation. The cracktip branch enrichment functions $F_{\alpha}(\mathbf{x})$ for isotropic materials are defined as [1]

$$
\begin{aligned}
& \left\{F_{\alpha}(\mathbf{x})\right\}_{\alpha=1}^{4} \\
& =\left\{\sqrt{r} \sin \frac{\theta}{2} \quad \sqrt{r} \cos \frac{\theta}{2} \sqrt{r} \sin \frac{\theta}{2} \sin \theta \quad \sqrt{r} \cos \frac{\theta}{2} \sin \theta\right\} .
\end{aligned}
$$

3.2. Discrete Equilibrium Equations. Considering a body $\Omega$ with an initial traction-free crack in the state of dynamic equilibrium, the weak form of the momentum equation is

$$
\int_{\Omega} \rho \ddot{\mathbf{u}} \cdot \delta \mathbf{u} d \Omega+\int_{\Omega} \boldsymbol{\sigma} \cdot \delta \boldsymbol{\varepsilon} d \Omega=\int_{\Omega} \mathbf{f} \cdot \delta \mathbf{u} d \Omega+\int_{\Gamma} \overline{\mathbf{f}} \cdot \delta \mathbf{u} d \Gamma
$$

where $\mathbf{u}$ is the displacement vector; $\boldsymbol{\sigma}$ and $\boldsymbol{\varepsilon}$ are the stress and strain tensors, respectively; $\mathbf{f}$ and $\overline{\mathbf{f}}$ are the body force and external traction vectors, respectively; and $\rho$ is the mass density.

The discretized form of (9) using the X-IGA approximation (3) can be written as

$$
\mathbf{M} \ddot{\boldsymbol{\delta}}+\mathbf{K} \boldsymbol{\delta}=\mathbf{F},
$$

where $\boldsymbol{\delta}=\left[\begin{array}{lll}\mathbf{u} & \mathbf{a} & \mathbf{b}\end{array}\right]^{T}$ is the vector of the unknown variable at control points and $\mathbf{M}, \mathbf{K}$, and $\mathbf{F}$ are, respectively, the global mass matrix, stiffness matrix, and external force vector at control points. 
The element contribution to $\mathbf{M}$ is

$$
\mathbf{m}_{i j}=\left[\begin{array}{lll}
\mathbf{m}_{i j}^{u u} & \mathbf{m}_{i j}^{u a} & \mathbf{m}_{i j}^{u b} \\
\mathbf{m}_{i j}^{a u} & \mathbf{m}_{i j}^{a a} & \mathbf{m}_{i j}^{a b} \\
\mathbf{m}_{i j}^{b u} & \mathbf{m}_{i j}^{b a} & \mathbf{m}_{i j}^{b b}
\end{array}\right],
$$

where

$$
\begin{gathered}
\mathbf{m}_{i j}^{u u}=\int_{\Omega} \rho\left(R_{i}\right)^{T} R_{j} d \Omega, \\
\mathbf{m}_{i j}^{a a}=\int_{\Omega} \rho\left(H(\mathbf{x})-H\left(\mathbf{x}_{i}\right)\right)\left(H(\mathbf{x})-H\left(\mathbf{x}_{j}\right)\right)\left(R_{i}\right)^{T} R_{j} d \Omega, \\
\mathbf{m}_{i j}^{b b}=\int_{\Omega} \rho\left(F_{\alpha}(\mathbf{x})-F_{\alpha}\left(\mathbf{x}_{i}\right)\right)\left(F_{\alpha}(\mathbf{x})-F_{\alpha}\left(\mathbf{x}_{j}\right)\right)\left(R_{i}\right)^{T} R_{j} d \Omega, \\
\mathbf{m}_{i j}^{u b}=\int_{\Omega} \rho\left(F_{\alpha}(\mathbf{x})-F_{\alpha}\left(\mathbf{x}_{j}\right)\right)\left(R_{i}\right)^{T} R_{j} d \Omega \quad(\alpha=1 \sim 4), \\
\mathbf{m}_{i j}^{a b}=\int_{\Omega}^{u a} \rho\left(H(\mathbf{x})-H\left(\mathbf{x}_{i}\right)\right)\left(F_{\alpha}(\mathbf{x})-F_{\alpha}\left(\mathbf{x}_{j}\right)\right)\left(R_{i}\right)^{T} R_{j} d \Omega
\end{gathered}
$$

and the element contribution to $\mathbf{K}$ is

$$
\mathbf{k}_{i j}=\left[\begin{array}{lll}
\mathbf{k}_{i j}^{u u} & \mathbf{k}_{i j}^{u a} & \mathbf{k}_{i j}^{u b} \\
\mathbf{k}_{i j}^{a u} & \mathbf{k}_{i j}^{a a} & \mathbf{k}_{i j}^{a b} \\
\mathbf{k}_{i j}^{b u} & \mathbf{k}_{i j}^{b a} & \mathbf{k}_{i j}^{b b}
\end{array}\right],
$$

where

$$
\mathbf{k}_{i j}^{r s}=\int_{\Omega_{e}}\left(\mathbf{B}_{i}^{r}\right)^{T} \mathbf{D B}_{j}^{s} d \Omega \quad(r, s=u, a, b)
$$

with

$$
\begin{aligned}
& \mathbf{B}_{i}^{u}=\left[\begin{array}{cc}
R_{i, x} & 0 \\
0 & R_{i, y} \\
R_{i, y} & R_{i, x}
\end{array}\right] \\
& \mathbf{B}_{i}^{a}=\left(H(\mathbf{x})-H\left(\mathbf{x}_{i}\right)\right)\left[\begin{array}{cc}
R_{i, x} & 0 \\
0 & R_{i, y} \\
R_{i, y} & R_{i, x}
\end{array}\right] \text {, } \\
& \mathbf{B}_{i}^{b}=\left[\begin{array}{llll}
\mathbf{B}_{i}^{b 1} & \mathbf{B}_{i}^{b 2} & \mathbf{B}_{i}^{b 3} & \mathbf{B}_{i}^{b 4}
\end{array}\right], \\
& \mathbf{B}_{i}^{b \alpha}=\left[\begin{array}{cc}
\left(R_{i}\left(F_{\alpha}(\mathbf{x})-F_{\alpha}\left(\mathbf{x}_{i}\right)\right)\right)_{, x} & 0 \\
0 & \left(R_{i}\left(F_{\alpha}(\mathbf{x})-F_{\alpha}\left(\mathbf{x}_{i}\right)\right)\right)_{, y} \\
\left(R_{i}\left(F_{\alpha}(\mathbf{x})-F_{\alpha}\left(\mathbf{x}_{i}\right)\right)\right)_{, y} & \left(R_{i}\left(F_{\alpha}(\mathbf{x})-F_{\alpha}\left(\mathbf{x}_{i}\right)\right)\right)_{, x}
\end{array}\right] \\
& (\alpha=1 \sim 4) \text {. }
\end{aligned}
$$

Additionally, the element contribution to $\mathbf{F}$ is

$$
\mathbf{f}_{i}=\left[\begin{array}{lll}
\mathbf{f}_{i}^{u} & \mathbf{f}_{i}^{a} & \mathbf{f}_{i}^{b}
\end{array}\right]
$$

with

$$
\begin{aligned}
\mathbf{f}_{i}^{u}= & \int_{\partial \Omega_{e}} R_{i} \overline{\mathbf{t}} d \Gamma+\int_{\Omega_{e}} R_{i} \mathbf{t} d \Omega, \\
\mathbf{f}_{i}^{a}= & \int_{\partial \Omega_{e}} R_{i}\left(H(\mathbf{x})-H\left(\mathbf{x}_{i}\right)\right) \overline{\mathbf{t}} d \Gamma \\
& +\int_{\Omega_{e}} R_{i}\left(H(\mathbf{x})-H\left(\mathbf{x}_{i}\right)\right) \mathbf{t} d \Omega \\
\mathbf{f}_{i}^{b \alpha}= & \int_{\partial \Omega_{e}} R_{i}\left(F_{\alpha}(\mathbf{x})-F_{\alpha}\left(\mathbf{x}_{i}\right)\right) \overline{\mathbf{t}} d \Gamma \\
& +\int_{\Omega_{e}} R_{i}\left(F_{\alpha}(\mathbf{x})-F_{\alpha}\left(\mathbf{x}_{i}\right)\right) \mathbf{t} d \Omega \quad(\alpha=1 \sim 4),
\end{aligned}
$$

where $\overline{\mathbf{t}}$ and $\mathbf{t}$ are the external traction and body force vectors, respectively.

3.3. Numerical Integration Scheme. The Gauss quadrature scheme is employed in the X-IGA. To obtain an accurate integration for crack tip elements and elements cut by crack, the triangular subdomain technique is used in the same way as that of the X-FEM [1]. For additional details, refer to [31].

3.4. Time Integration Scheme. The Newmark method is adopted for the time integration of (10). At time step $n$, the discrete simultaneous equations are described as

$$
\begin{array}{r}
\left(\mathbf{M}+\beta \Delta t^{2} \mathbf{K}\right) \ddot{\boldsymbol{\delta}}_{n}=\mathbf{F}_{n}-\mathbf{K}\left[\boldsymbol{\delta}_{n-1}+\Delta t \dot{\boldsymbol{\delta}}_{n-1}\right. \\
\left.+(1-2 \beta) \frac{\Delta t^{2}}{2} \ddot{\boldsymbol{\delta}}_{n-1}\right], \\
\dot{\boldsymbol{\delta}}_{n}=\dot{\boldsymbol{\delta}}_{n-1}+\left[(1-\alpha) \ddot{\boldsymbol{\delta}}_{n-1}+\alpha \ddot{\boldsymbol{\delta}}_{n}\right] \Delta t, \\
\boldsymbol{\delta}_{n}=\boldsymbol{\delta}_{n-1}+\dot{\boldsymbol{\delta}}_{n-1} \Delta t \\
+\left[\left(\frac{1}{2}-\beta\right) \ddot{\boldsymbol{\delta}}_{n-1}+\beta \ddot{\boldsymbol{\delta}}_{n}\right] \Delta t^{2},
\end{array}
$$

where $\Delta t$ is the time step and the unconditionally stable conditions are $\alpha \geq 0.5$ and $\beta \geq 0.25(\alpha+0.5)^{2}$. In this study, $\alpha=0.5$ and $\beta=0.25$ are chosen.

The implementation procedure of the Newmark time integration scheme applying into the X-IGA is outlined as follows.

(1) The initial velocities and initial displacements are set to be zero; then the initial accelerations are obtained with (10).

(2) Compute the constants $a_{0}=1 / \beta \Delta t^{2}, a_{1}=\alpha / \beta \Delta t$, $a_{2}=1 / \beta \Delta t, a_{3}=(1 / 2 \beta)-1, a_{4}=(\alpha / \beta)-1$, $a_{5}=(\Delta t / 2)((\alpha / \beta)-2), a_{6}=\Delta t(1-\alpha)$, and $a_{7}=\alpha \Delta t$.

(3) Compute $\overline{\mathbf{K}}=a_{0} \mathbf{M}+\mathbf{K}$. 
(4) Loop over the time steps as follows:

(a) compute $\overline{\mathbf{F}_{n}}=\mathbf{F}_{n}+\mathbf{M}\left(a_{0} \boldsymbol{\delta}_{n-1}+a_{2} \dot{\boldsymbol{\delta}}_{n-1}+a_{3} \ddot{\boldsymbol{\delta}}_{n-1}\right)$;

(b) compute the displacements $\boldsymbol{\delta}_{n}$ by solving $\overline{\mathbf{K}} \boldsymbol{\delta}_{n}=$ $\overline{\mathbf{F}}_{n}$;

(c) solve $\ddot{\boldsymbol{\delta}}_{n}=a_{0}\left(\boldsymbol{\delta}_{n}-\boldsymbol{\delta}_{n-1}\right)-a_{2} \dot{\boldsymbol{\delta}}_{n-1}-a_{3} \ddot{\boldsymbol{\delta}}_{n-1}$ for the value $\ddot{\boldsymbol{\delta}}_{n}$, and the velocities $\dot{\boldsymbol{\delta}}_{n}$ are calculated by using $\dot{\boldsymbol{\delta}}_{n}=\dot{\boldsymbol{\delta}}_{n-1}+a_{6} \ddot{\boldsymbol{\delta}}_{n-1}+a_{7} \ddot{\boldsymbol{\delta}}_{n}$.

(5) Repeat the loop for the next time step until the maximum time step is reached.

\section{DSIF Calculations}

The DSIFs are evaluated by using the domain form of the contour interaction integral technique. The following states are considered: state $1\left(\sigma_{i j}^{(1)}, \varepsilon_{i j}^{(1)}, u_{i}^{(1)}\right)$, which corresponds to the actual state; state $2\left(\sigma_{i j}^{(2)}, \varepsilon_{i j}^{(2)}, u_{i}^{(2)}\right)$, which is an auxiliary state that will be selected as the asymptotic field for model I or II. The interaction integral may be written as [37]

$$
\begin{aligned}
I^{(1,2)}= & \int_{A}\left[-W^{(1,2)} \delta_{1 j}+\sigma_{i j}^{(1)} \frac{\partial u_{i}^{(2)}}{\partial x_{1}}+\sigma_{i j}^{(2)} \frac{\partial u_{i}^{(1)}}{\partial x_{1}}\right] \frac{\partial q}{\partial x_{j}} d A \\
& +\int_{A} \rho \ddot{u}^{(1)} \frac{\partial u_{i}^{(2)}}{\partial x_{1}} d A,
\end{aligned}
$$

where $W^{(1,2)}=\sigma_{i j}^{(1)} \varepsilon_{i j}^{(2)}=\sigma_{i j}^{(2)} \varepsilon_{i j}^{(1)}$ is the interaction strain energy and $q$ is a weighing function. The second term of the right-hand side of the equation is the contribution of the inertia forces.

For isotropic materials, the relation between the interaction integral $I^{(1,2)}$ and the stress intensity factors is expressed as [37]

$$
I^{(1,2)}=\frac{2\left(K_{I}^{(1)} K_{I}^{(2)}+K_{I I}^{(1)} K_{I I}^{(2)}\right)}{E^{*}},
$$

where $E^{*}=E$ is for plane stress and $E^{*}=E /\left(1-\nu^{2}\right)$ is for plane strain.

The relation between the interaction integral $I^{(1,2)}$ and the stress intensity factors for orthotropic materials is expressed as [36]

$$
\begin{aligned}
I^{(1,2)}= & 2 c_{11} K_{I}^{(1)} K_{I}^{(2)}+c_{12}\left(K_{I}^{(1)} K_{I I}^{(2)}+K_{I}^{(2)} K_{I I}^{(1)}\right) \\
& +2 c_{22} K_{I I}^{(1)} K_{I I}^{(2)}
\end{aligned}
$$

with

$$
\begin{aligned}
& c_{11}=-\frac{a_{22}}{2} \operatorname{Im}\left(\frac{\mu_{1}+\mu_{2}}{\mu_{1} \mu_{2}}\right), \\
& c_{12}=-\frac{a_{22}}{2} \operatorname{Im}\left(\frac{1}{\mu_{1} \mu_{2}}\right)+\frac{a_{11}}{2} \operatorname{Im}\left(\mu_{1} \mu_{2}\right), \\
& c_{22}=\frac{a_{11}}{2} \operatorname{Im}\left(\mu_{1}+\mu_{2}\right) .
\end{aligned}
$$

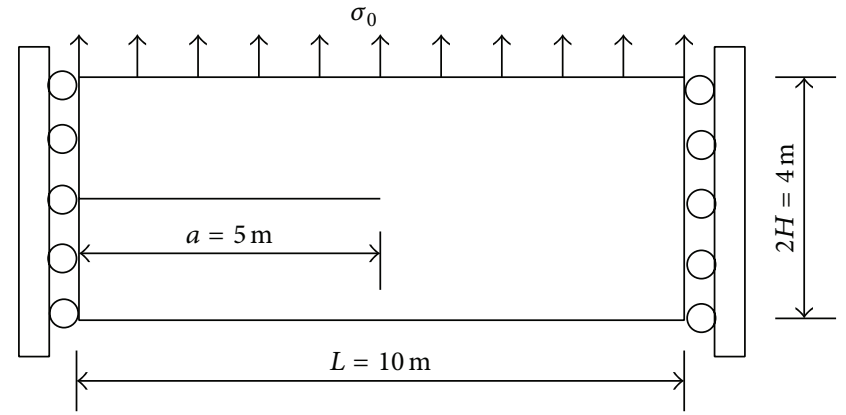

Figure 1: Geometry and loading of a semi-infinite crack.

Choosing state 2 as mode I or mode II leads to mode-I or mode-II DSIF in terms of the interaction integral.

For isotropic materials,

$$
\left\{\begin{array}{l}
K_{I}^{(1)} \\
K_{I I}^{(1)}
\end{array}\right\}=\frac{E^{*}}{2}\left\{\begin{array}{c}
I^{(1, I)} \\
I^{(1, I I)}
\end{array}\right\} .
$$

For orthotropic materials,

$$
\left\{\begin{array}{l}
K_{I}^{(1)} \\
K_{I I}^{(1)}
\end{array}\right\}=\left[\begin{array}{cc}
2 c_{11} & c_{12} \\
c_{12} & 2 c_{22}
\end{array}\right]^{-1}\left\{\begin{array}{l}
I^{(1, I)} \\
I^{(1, I I)}
\end{array}\right\} .
$$

\section{Numerical Simulations}

In this section, several examples of stationary cracks in isotropic/orthotropic media with available reference solutions are investigated to assess the accuracy of the proposed approach. The first two examples demonstrate the efficiency of the X-IGA for isotropic material problems, and the next two illustrate the application of the X-IGA to orthotropic material problems, whereas the last example demonstrates the capability and versatility of the X-IGA in modeling the complicated geometries. In all examples, the Heaviside step loading is considered and degree $3 \mathrm{X}-\mathrm{IGA}$ is adopted; that is, $p=q=3$. Meshes are generated with linear parameterization method. In the case of investigating the effects of different meshes on the results, different uniform meshes are adopted; in other cases, the fine meshes are used around the crack, while the coarse meshes are used in other domains; thus excellent accuracy can be achieved at a low cost.

5.1. Semi-Infinite Crack in an Infinite Isotropic Plate. For the first example, we consider an infinite isotropic plate with a semi-infinite crack subjected to a tensile stress wave perpendicular to the crack face, as shown in Figure 1. The material properties of the media are as follows: Young's modulus $E=210 \mathrm{GPa}$, Poisson's ratio $v=0.3$, and mass density $\rho=8000 \mathrm{~kg} / \mathrm{m}^{3}$. The tensile stress $\sigma_{0}=500 \mathrm{MPa}$. 


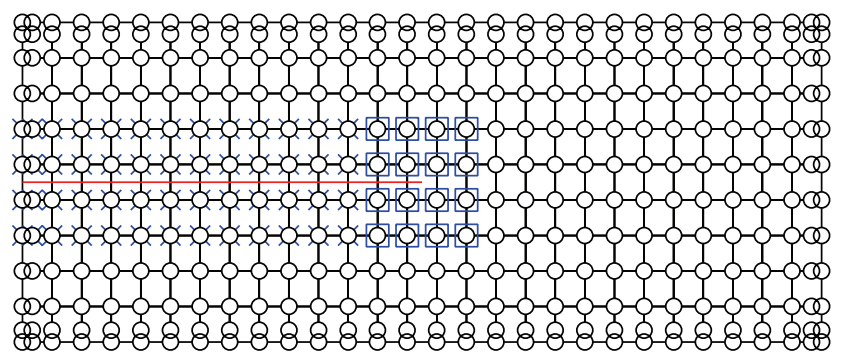

FIGURE 2: Control points and elements for degree 3 X-IGA with 27 $\times 9$ elements (the blue cross represents the control points enriched by Heaviside function; the blue square represents the control points enriched by crack tip enrichment functions; the red line represents the crack).

The theoretical solution of mode-I DSIF for the stationary crack is expressed as [38]

$$
K_{I}^{\mathrm{dyn}}(t)= \begin{cases}0, & t<t_{c} \\ \frac{2 \sigma_{0}}{1-v} \sqrt{\frac{c_{d}\left(t-t_{c}\right)(1-2 v)}{\pi}}, & t_{c} \leq t \leq 3 t_{c},\end{cases}
$$

where $t_{c}=\left(H / c_{d}\right)\left(c_{d}\right.$ : the dilatational wave speed $)$.

5.1.1. Convergence Study of the DSIF versus Meshes. In this study, five meshes with $27 \times 9,47 \times 17,67 \times 25,87 \times 33$, and 107 $\times 41$ elements are considered. Figure 2 illustrates the typical regular mesh of $27 \times 9$ elements.

Figure 3 presents the normalized mode-I DSIFs as a function of time for the five considered meshes. In the mesh refinement process, the mode-I DSIFs obtained by the X-IGA converge well to the theoretical solution. The maximum error increases beside $t=t_{c}$ for the coarse mesh and decreases significantly for the fine mesh. Similar observation was noted in the simulation of the singular edge-based smoothed finite element method (sES-FEM) [37]. The same simulations were conducted by other researches by using different methods. However, the same conclusion is recorded; that is, the error is lower in later stages than in earlier stages. The relative errors during $1.5 t_{c} \leq t \leq 3 t_{c}$ are presented in Figure 4 , in which the error is unchanged as the mesh is refined to a certain extent. In this study, the errors are the same for meshes with $67 \times 25$, $87 \times 33$, and $107 \times 41$ elements.

5.1.2. Comparison with X-FEM Results. The X-IGA results using $67 \times 25$ mesh elements are compared with three available X-FEM solutions, including the X-FEM using mass lumping $(120 \times 60$ quadrilateral elements) [39], the meshfree enriched X-FEM with crack-tip enrichments $(78 \times$ 39 quadrilateral elements) [38], and X-FEM with a new enrichment function $(120 \times 59$ quadrilateral elements) [40]. Figure 5 shows the comparative study of the normalized mode-I DSIFs. The results obtained with X-FEM using mass lumping [39] are unstable, and the accuracy of this method is the worst recorded among the four approaches. The results obtained with the X-IGA, the X-FEM using an enrichment

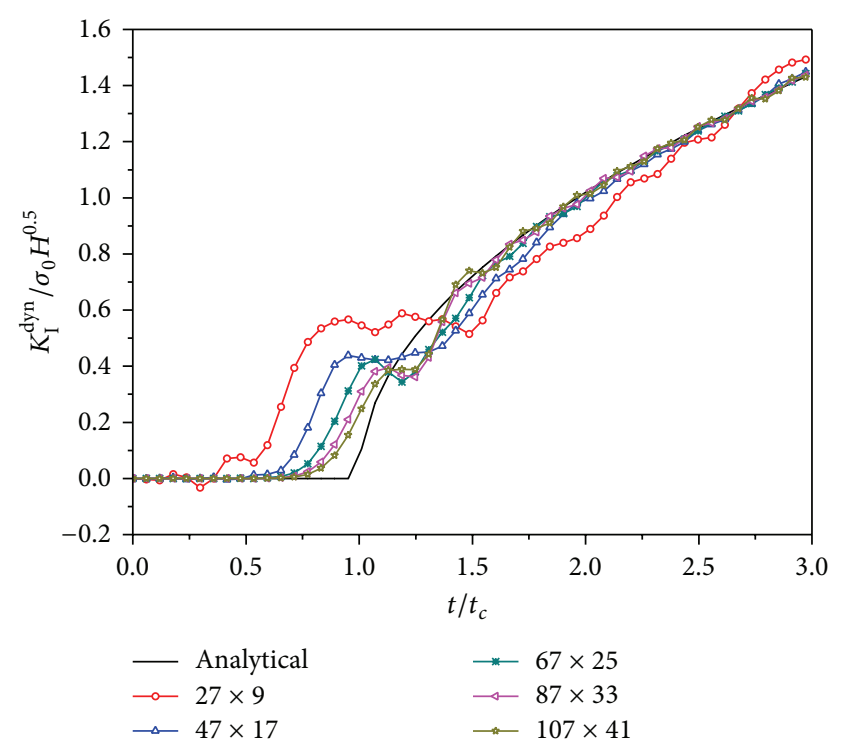

FIgURE 3: Convergence of the normalized mode-I DSIFs versus mesh densities.

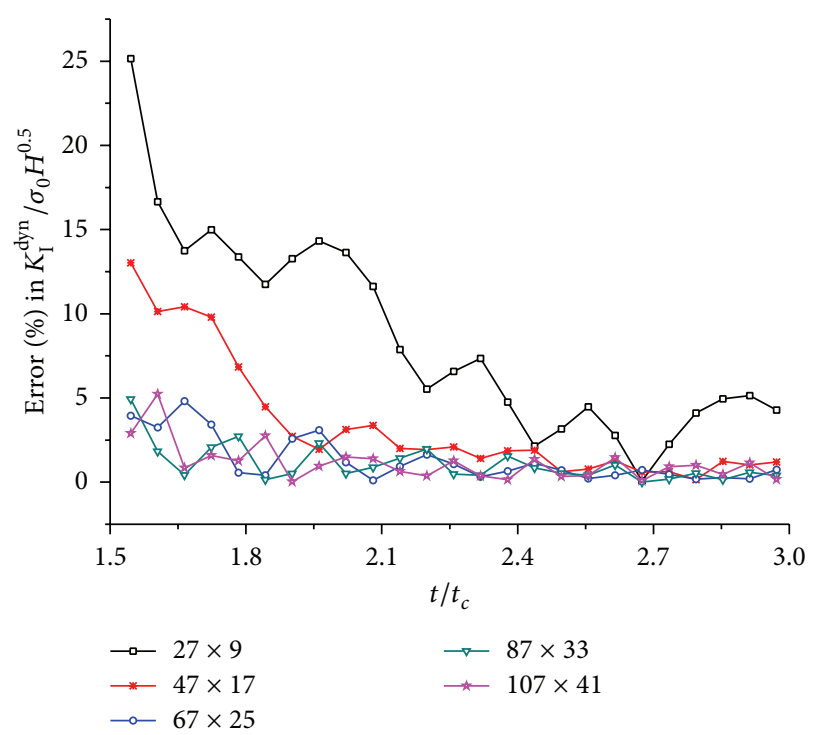

FIGURE 4: Convergence in percentage errors for the normalized mode-I DSIFs versus mesh densities during $1.5 t_{c} \leq t \leq 3 t_{c}$.

function, and the mesh-free enriched X-FEM with cracktip enrichments are very accurate during $1.5 t_{c} \leq t \leq 3 t_{c}$; however, the results are relatively poor during $t \leq 1.5 t_{c}$. The $\mathrm{X}$-IGA results are more accurate than other numerical results during $1.5 t_{c} \leq t \leq 3 t_{c}$.

5.2. Arbitrarily Oriented Central Crack in a Rectangular Isotropic Plate. We consider a rectangular isotropic plate with an arbitrarily oriented central crack subjected on the top and bottom of a uniform impact loading, as shown in Figure 6(a). The length of the crack is $2 a=4.8 \mathrm{~mm}$. The material properties of the plate are as follows: Young's modulus $E=200 \mathrm{GPa}$, Poisson's ratio $v=0.3$, and mass 


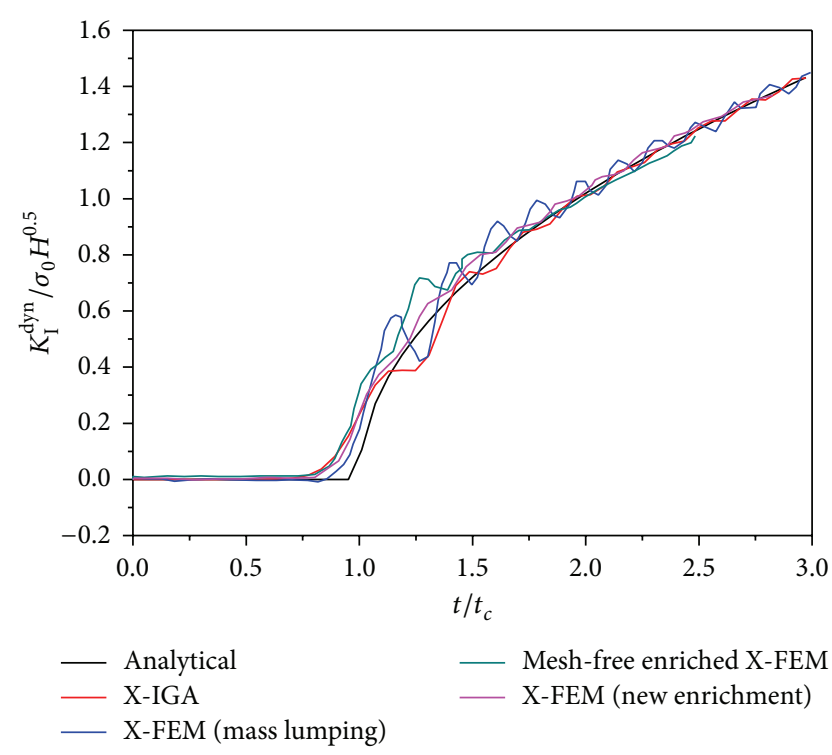

FIGURE 5: Comparison of the normalized mode-I DSIFs among different methods.

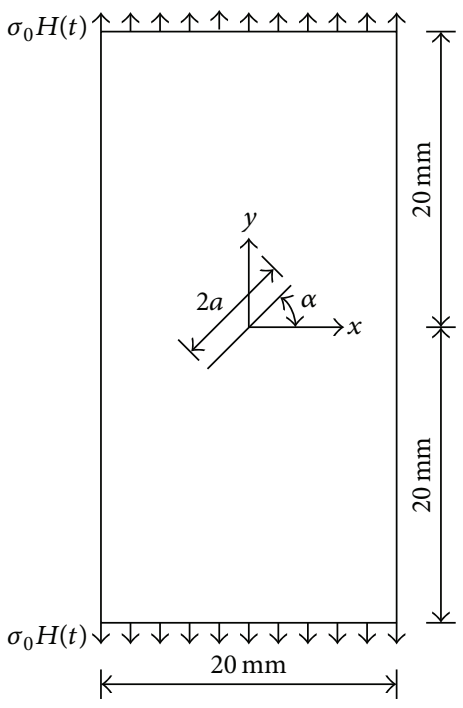

(a)

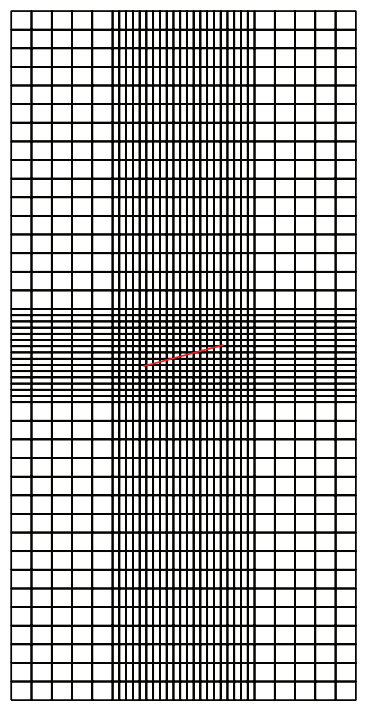

(b)
FIGURE 6: A rectangular plate with an arbitrarily oriented crack. (a) Geometry and loading, (b) computational meshes.

density $\rho=5000 \mathrm{~kg} / \mathrm{m}^{3}$. Three crack inclination angles are investigated, and the total time of the simulation is $20 \mu \mathrm{s}$. In the X-IGA, the computational mesh is independent of the crack; therefore, the computation meshes for the three crack inclination angles are the same, as shown in Figure 6(b).

Figures 7 and 8 , respectively, present the normalized mode-I and mode-II DSIFs for the three considered crack angles computed by the X-IGA, sES-FEM [37], and X-FEM. Figure 6(b) is also the mesh for the X-FEM, whereas the mesh of $60 \times 120$ triangular elements is used in the analysis of the sES-FEM.

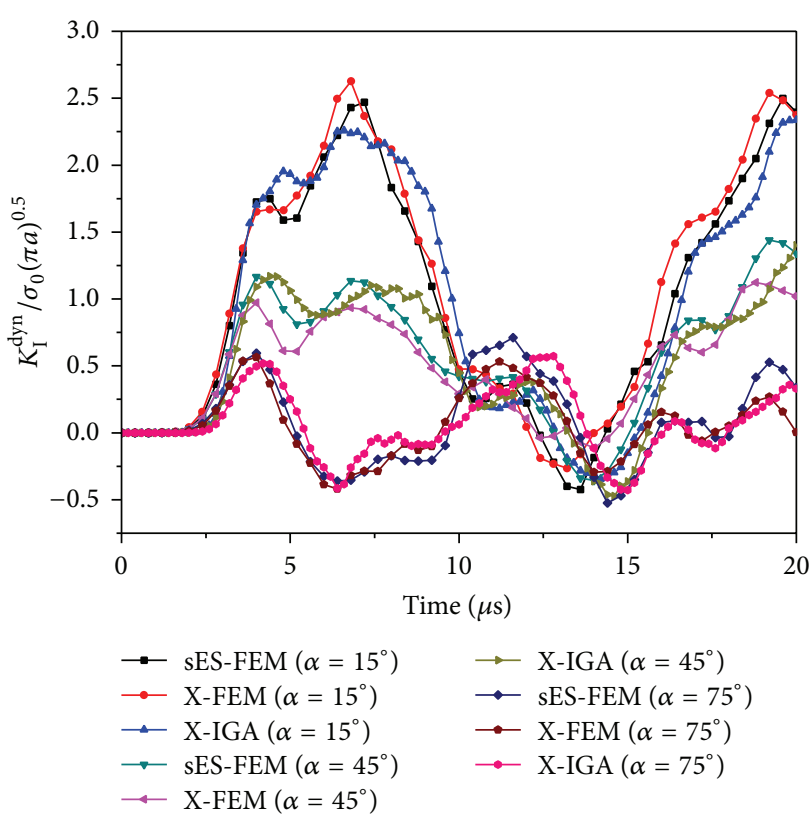

FIGURE 7: Comparison of the normalized mode-I DSIFs obtained by the X-FEM, the sES-FEM, and the X-IGA for three crack inclination angles.

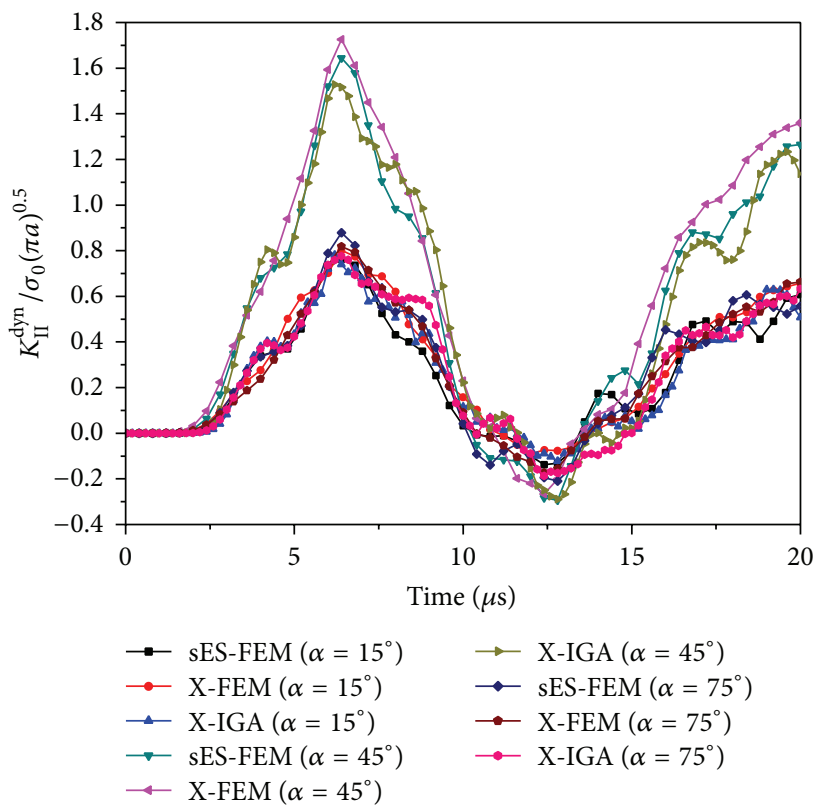

FIgURE 8: Comparison of the normalized mode-II DSIFs obtained by the X-FEM, the sES-FEM, and the X-IGA for three crack inclination angles.

A good agreement can be observed for the three considered crack inclination angles among the three numerical approaches.

5.3. Edge Crack in a Rectangular Orthotropic Plate. As a third example, a rectangular orthotropic plate with a $12 \mathrm{~mm}$ edge horizontal crack is considered (Figure 9). The plane stress 


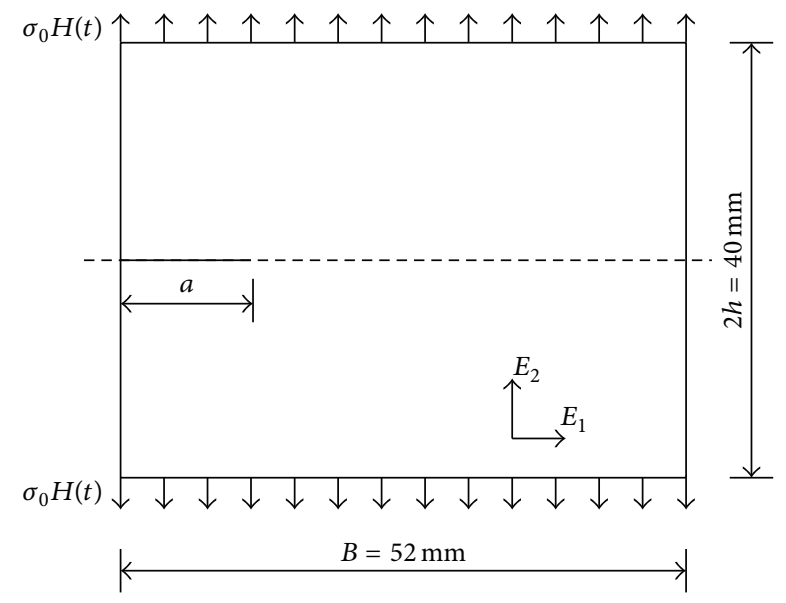

FIGURE 9: Geometry and loads of an edge crack in a rectangular orthotropic plate.

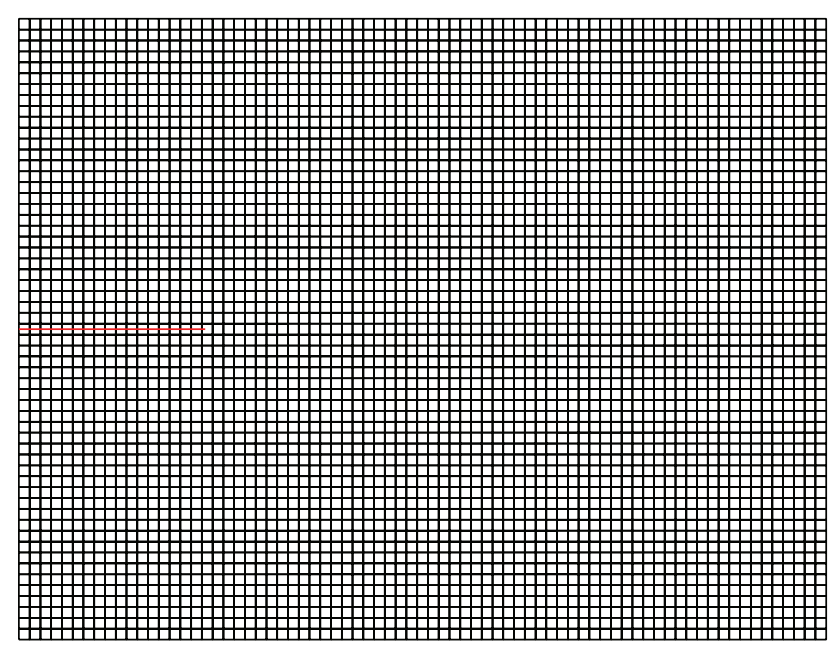

Figure 10: The X-IGA discretization for an edge crack in a rectangular orthotropic plate.

condition is considered, and the material properties of the plate are as follows: $E_{1}=118.3 \mathrm{GPa}, E_{2}=54.8 \mathrm{GPa}, G_{12}=$ $8.79 \mathrm{GPa}, v_{12}=0.083$, and $\rho=1900 \mathrm{~kg} / \mathrm{m}^{3}$. The time step $\Delta t=$ $\left(a / 10 c_{L}\right)$ is selected, where $c_{L}$ is the wave velocity along the $E_{2}$ material-axis.

Figure 10 shows the mesh with $75 \times 57$ elements used in the simulation. The normalized mode-I DSIFs achieved with the X-IGA (Figure 11) were compared with the reference boundary element method (BEM) (21 elements for the external boundary and eight elements for the crack) [41], the X-FEM $(78 \times 60$ quadrilateral elements) [42], and the conventional finite element method (FEM) results (via ANSYS) [41]. Good agreement is observed among the methods. The X-IGA results more closely match the FEM and the BEM solution compared with the X-FEM results. In [42], the DSIFs are evaluated by using the domain separation integral method, which could be the reason that the X-FEM results are different.

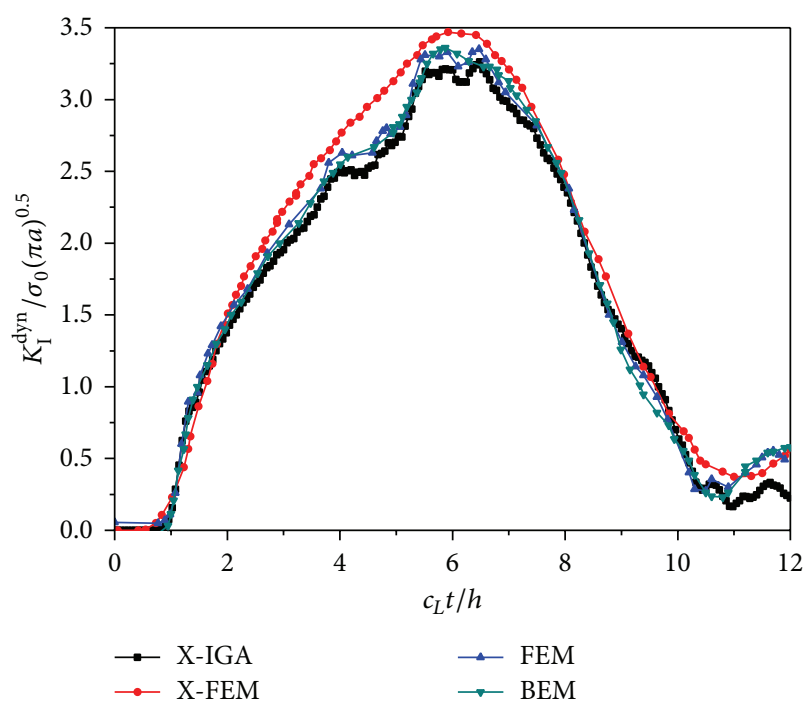

FIGURE 11: Comparison of the normalized mode-I DSIFs among different methods.

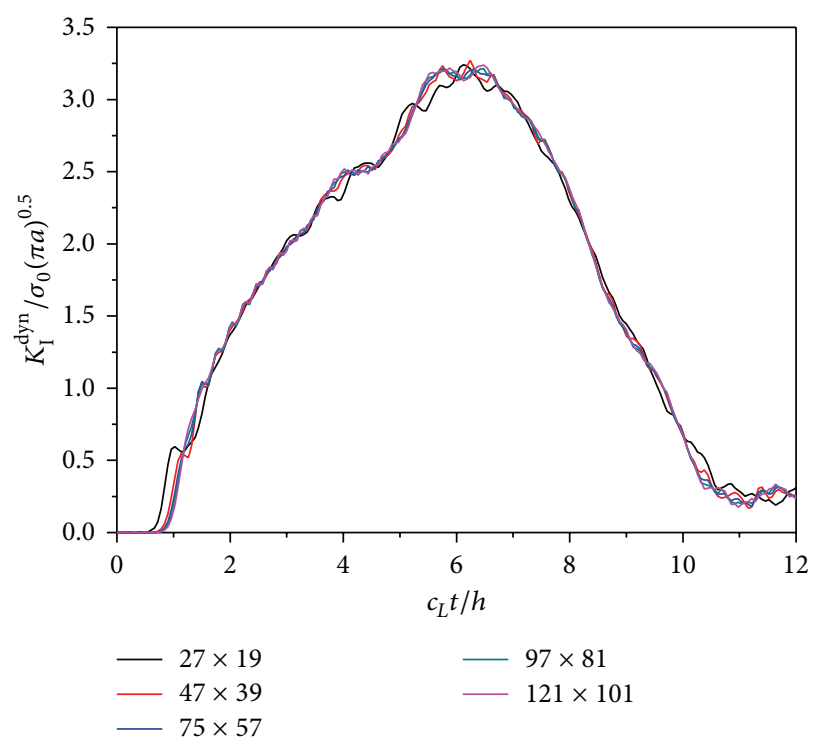

FIGURE 12: Comparison of the normalized mode-I DSIFs for different meshes.

To analyze the effects of different meshes on DSIFs, five different meshes with $27 \times 19,47 \times 39,75 \times 57,97 \times 81$, and $121 \times 101$ elements are adopted to simulate the problem. The results are shown in Figure 12. Accurate results can be obtained by using the X-IGA with a relatively coarse mesh.

5.4. Central Crack with Different Orientations of the Axes of Orthotropy. A rectangular orthotropic plate with a single central horizontal crack was subjected to a Heaviside step tensile distributed load, as shown in Figure 13(a). The length of the crack is $2 a=4.8 \mathrm{~mm}$ and $h=20 \mathrm{~mm}$. The material properties of the plate are the same as those in 


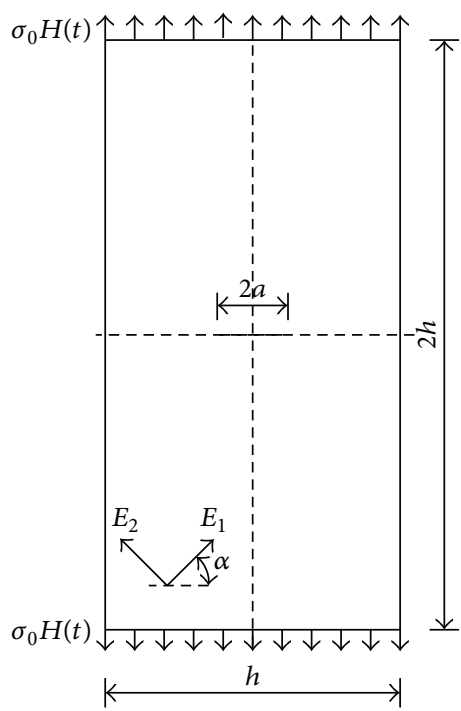

(a)

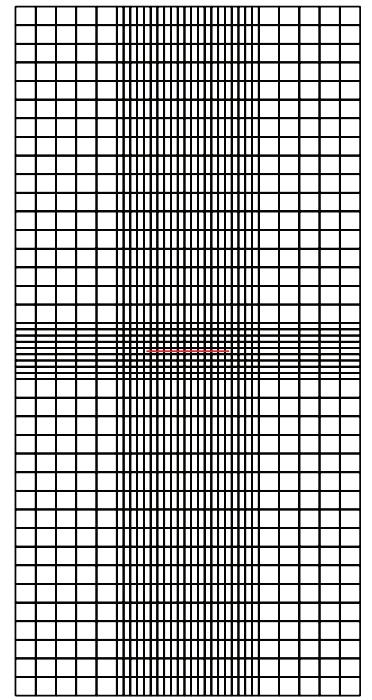

(b)
FIGURE 13: A single central crack in a rectangular orthotropic plate. (a) Geometry and loadings, (b) computational meshes.

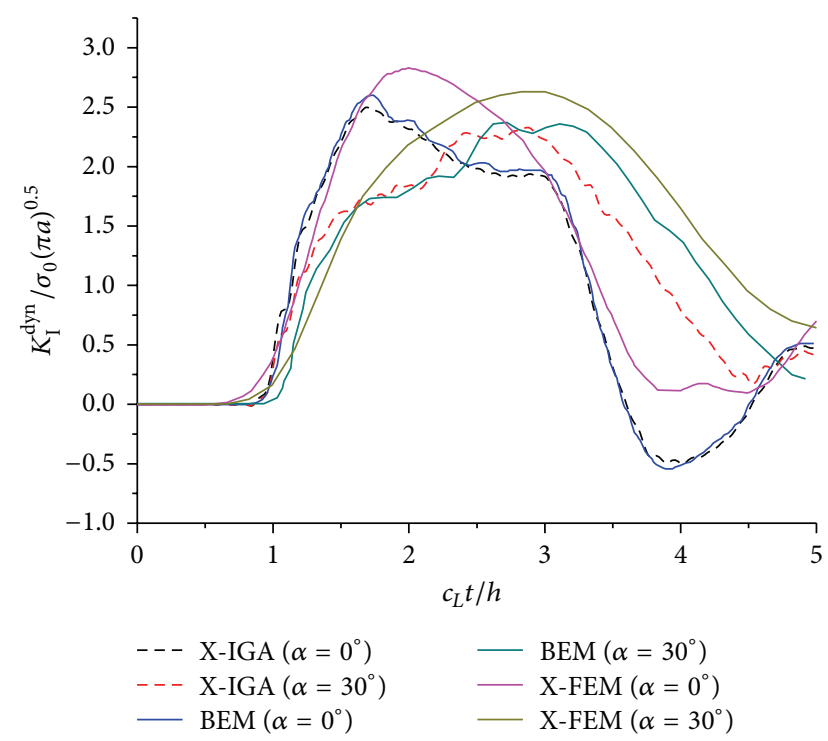

FIGURE 14: Normalized mode-I DSIFs for a single central crack in a rectangular plate.

the example in Section 5.3. The plane stress state is assumed, and the time step $\Delta t=\left(h / 50 c_{L}\right)$ is selected.

Two different inclination angles between the $E_{1}$ materialaxis and the crack-face are considered. Figures 14 and 15 show the comparison of the numerical results of BEM (24 elements for the external boundary and 10 for the crack) [41] and XFEM (50 × 100 quadrilateral elements) [42]. General trends are in good agreement across all methods; the X-IGA results more closely match the BEM solution compared with the $\mathrm{X}$ FEM; the reason for this phenomenon is the same as the above example.

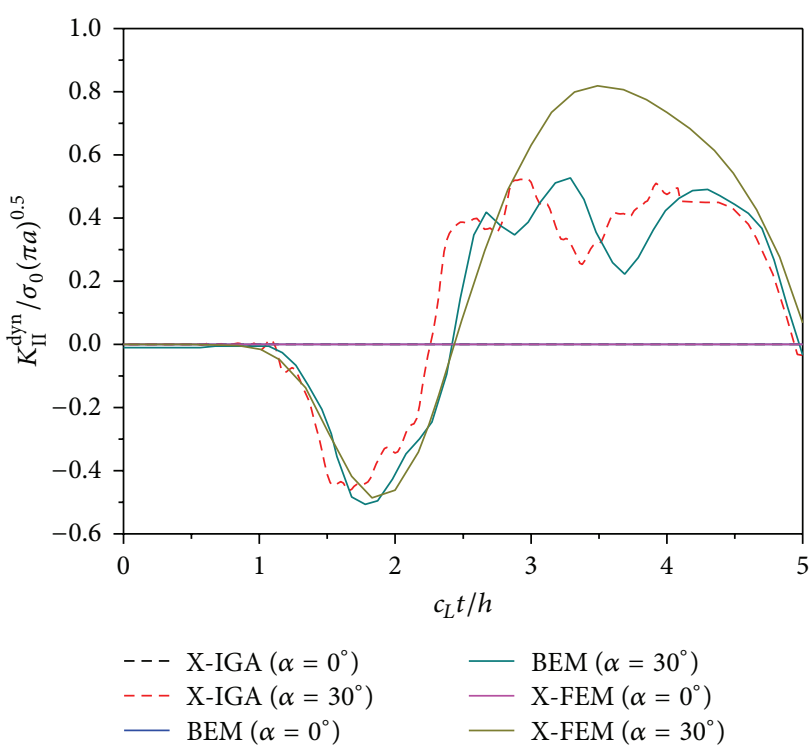

FIGURE 15: Normalized mode-II DSIFs for a single central crack in a rectangular plate.

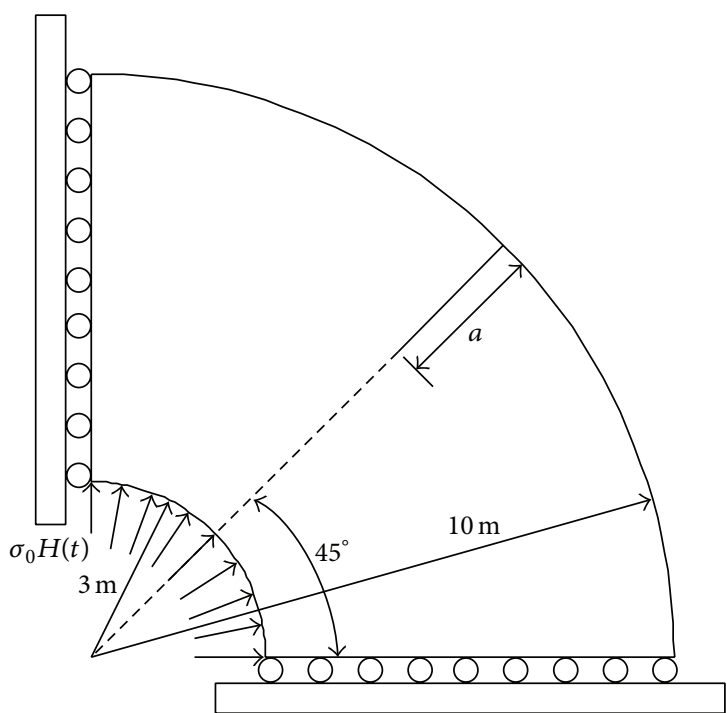

FIGURE 16: Geometry and loads of an edge crack in an annular isotropic plate.

5.5. Edge Crack in an Annular Isotropic Plate. To illustrate the capability and versatility of the X-IGA in modeling the complicated geometries, the last example deals with an annular isotropic plate under a uniform pressure. The geometry and loads of the plate are depicted in Figure 16. The length of the crack is $a=2 \mathrm{~m}$; the material properties of the plate are as follows: Young's modulus $E=210 \mathrm{GPa}$, Poisson's ratio $\nu=0.3$, and mass density $\rho=8000 \mathrm{~kg} / \mathrm{m}^{3}$. The total time of the simulation is $0.004 \mathrm{~s}$. The plane strain state is assumed, and the time step $\Delta t=8 \times 10^{-5} \mathrm{~s}$ is selected.

The meshes are depicted in Figure 17 for 285 control points and 221 elements. To perform a comparison, this 


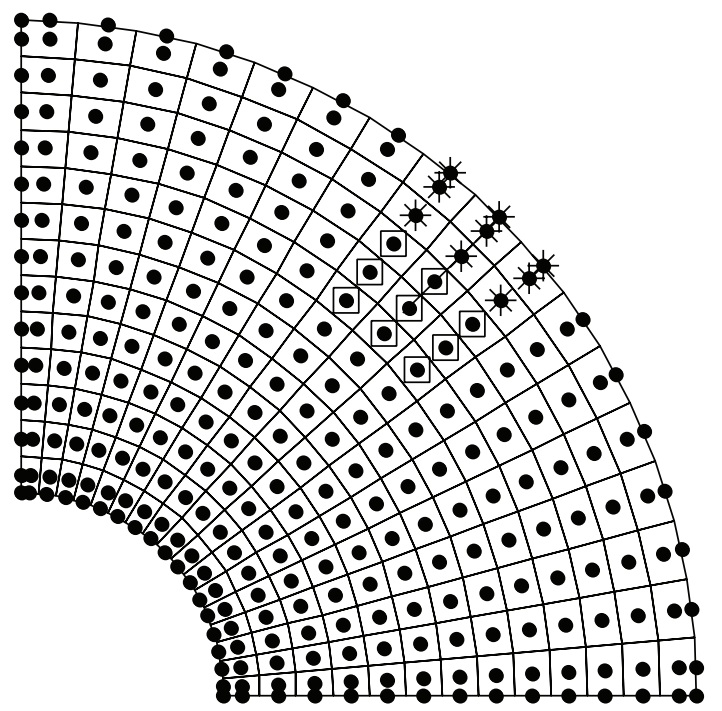

FIGURE 17: Meshes and control points. (The cross represents the control points enriched by Heaviside function; the square represents the control points enriched by crack tip enrichment functions.)

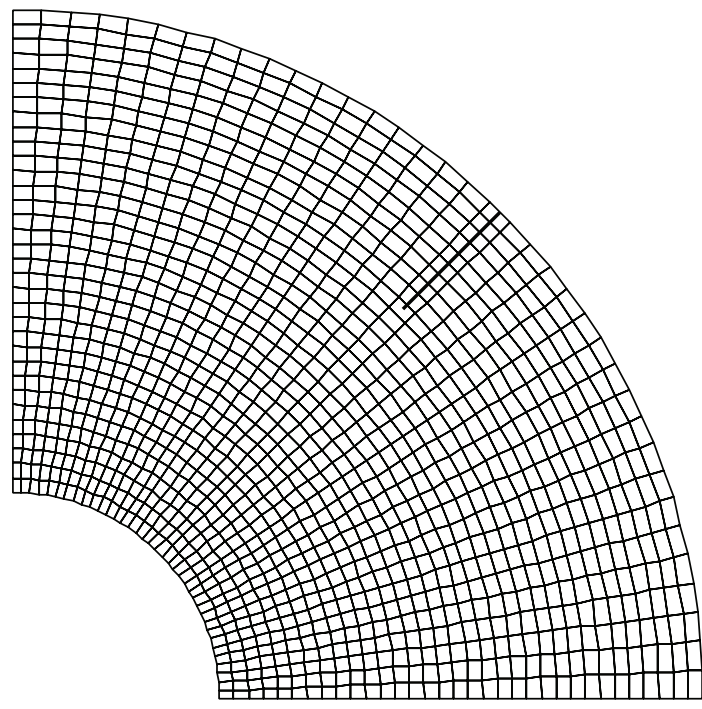

FIGURE 18: The X-FEM meshes.

example is solved by the X-FEM, and the X-FEM meshes are shown in Figure 18 for 1292 nodes and 1221 quadrilateral elements.

The DSIFs are computed with the X-FEM and the XIGA, and the results are shown in Figure 19. It is found that the results from the X-IGA with a relatively coarse mesh are in good agreement with those from the X-FEM with a very fine mesh. The amplitude of the normalized mode-I DSIF is negative in some time ranges, which may be due to the contact between two crack-faces which is not taken into consideration in the present study.

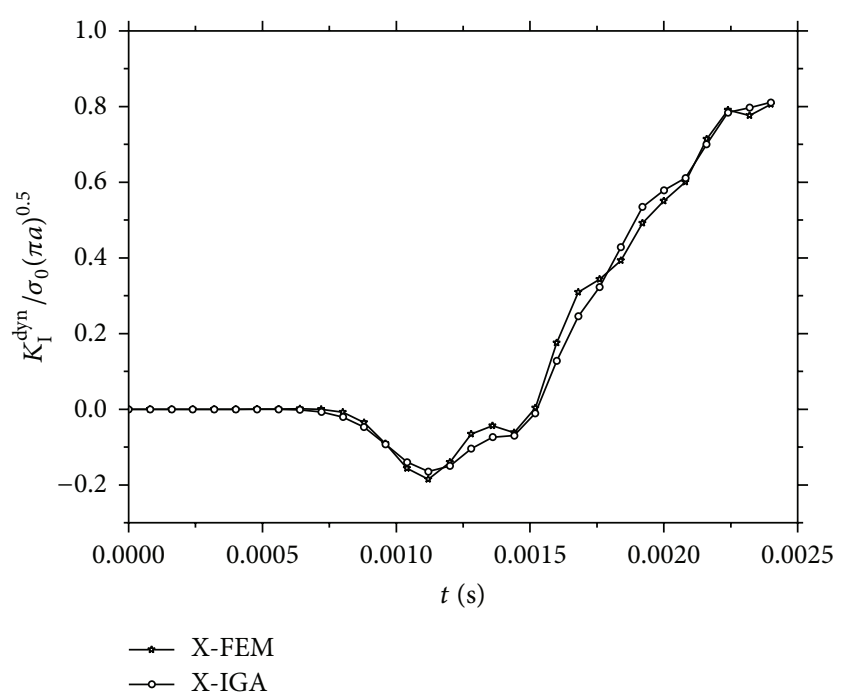

FIGURE 19: Comparison of the normalized mode-I DSIFs between the X-FEM and the X-IGA.

\section{Conclusions and Prospects}

The NURBS-based extended isogeometric analysis (X-IGA) was extended to investigate the dynamic fracture behavior of stationary cracks in isotropic/orthotropic media. A corresponding dynamic X-IGA model was developed, the Newmark time integration scheme was used to achieve the dynamic response, and the DSIFs were evaluated with the contour interaction integral technique. Numerical results indicate that accurate results can be obtained by using the $\mathrm{X}$ IGA with a relatively coarse mesh, and the X-IGA is suitable to model the cracked complicated geometries.

The NURBS-based isogeometric analysis has several drawbacks including the handling trimmed geometries and the local refinement. The isogeometric analysis based on Tsplines can effectively overcome these drawbacks [43]. A key advantage of the extended isogeometric analysis over isogeometric analysis is the ability to model crack propagation. Therefore, the application of extended isogeometric analysis based on T-splines to dynamic crack propagation analysis in isotropic/orthotropic media is an area of considerable promise. On the other hand, the smoothed finite element method (SFEM) developed by combining the smoothing technique with the finite element method is an efficient and accurate numerical simulation tool for the dynamic fracture problems [44]; therefore combining the smoothing technique with the X-IGA for fracture analysis is another area of considerable promise.

\section{Conflict of Interests}

The authors declare that there is no conflict of interests regarding the publication of this paper. 


\section{Acknowledgment}

This work is supported by the National Natural Science Foundation of China (51179063). The financial support is gratefully acknowledged.

\section{References}

[1] T. Belytschko and T. Black, "Elastic crack growth in finite elements with minimal remeshing," International Journal for Numerical Methods in Engineering, vol. 45, no. 5, pp. 601-620, 1999.

[2] N. Moës, J. Dolbow, and T. Belytschko, "A finite element method for crack growth without remeshing," International Journal for Numerical Methods in Engineering, vol. 46, no. 1, pp. 131-150, 1999.

[3] X. Y. Liu, Q. Z. Xiao, and B. L. Karihaloo, "XFEM for direct evaluation of mixed mode SIFs in homogeneous and bi-materials," International Journal for Numerical Methods in Engineering, vol. 59, no. 8, pp. 1103-1118, 2004.

[4] T. T. Yu and P. Liu, "Improved implementation of the extended finite element method for stress analysis around cracks," Archives of Civil and Mechanical Engineering, vol. 11, no. 3, pp. 787-805, 2011.

[5] J. V. Cox, "An extended finite element method with analytical enrichment for cohesive crack modeling," International Journal for Numerical Methods in Engineering, vol. 78, no. 1, pp. 48-83, 2009.

[6] A. R. Khoei and K. Karimi, "An enriched-FEM model for simulation of localization phenomenon in Cosserat continuum theory," Computational Materials Science, vol. 44, no. 2, pp. 733749,2008

[7] R. Duddu, S. Bordas, D. Chopp, and B. A. Moran, "A combined extended finite element and level set method for biofilm growth," International Journal for Numerical Methods in Engineering, vol. 74, no. 5, pp. 848-870, 2008.

[8] J. Chessa, P. Smolinski, and T. Belytschko, "The extended finite element method (XFEM) for solidification problems," International Journal for Numerical Methods in Engineering, vol. 53, no. 8, pp. 1959-1977, 2002.

[9] S. Groß and A. Reusken, "An extended pressure finite element space for two-phase incompressible flows with surface tension," Journal of Computational Physics, vol. 224, no. 1, pp. 40-58, 2007.

[10] P. Liu, T. T. Yu, T. Q. Bui, and C. Zhang, “Transient dynamic crack analysis in non-homogeneous functionally graded piezoelectric materials by the X-FEM," Computational Materials Science, vol. 69, pp. 542-558, 2013.

[11] T. T. Yu and L. Y. Shi, "Determination of sharp V-notch stress intensity factors using the extended finite element method," The Journal of Strain Analysis for Engineering Design, vol. 47, no. 2, pp. 95-103, 2012.

[12] T. T. Yu, "The extended finite element method (XFEM) for discontinuous rock masses," Engineering Computations, vol. 28, no. 3, pp. 340-369, 2011.

[13] L. Chen, T. Rabczuk, G. R. Liu, S. Bordas, K. Y. Zeng, and P. Kerfriden, "Extended finite element method with edge-based strain smoothing (ESm-XFEM) for linear elastic crack growth," Computer Methods in Applied Mechanics and Engineering, vol. 209-212, pp. 250-265, 2012.
[14] N. Sukumar, D. L. Chopp, N. Moës, and T. Belytschko, "Modeling holes and inclusions by level sets in the extended finiteelement method," Computer Methods in Applied Mechanics and Engineering, vol. 190, no. 46-47, pp. 6183-6200, 2001.

[15] J. Réthoré, A. Gravouil, and A. Combescure, "An energy-conserving scheme for dynamic crack growth using the extended finite element method," International Journal for Numerical Methods in Engineering, vol. 63, no. 5, pp. 631-659, 2005.

[16] T. J. R. Hughes, J. A. Cottrell, and Y. Bazilevs, "Isogeometric analysis: CAD, finite elements, NURBS, exact geometry and mesh refinement," Computer Methods in Applied Mechanics and Engineering, vol. 194, no. 39-41, pp. 4135-4195, 2005.

[17] R. Bouclier, T. Elguedj, and A. Combescure, "Locking free isogeometric formulations of curved thick beams," Computer Methods in Applied Mechanics and Engineering, vol. 245-246, pp. 144-162, 2012.

[18] D. J. Benson, Y. Bazilevs, M. C. Hsu, and T. J. R. Hughes, "Isogeometric shell analysis: the Reissner-Mindlin shell," Computer Methods in Applied Mechanics and Engineering, vol. 199, no. 5-8, pp. 276-289, 2010.

[19] C. H. Thai, H. Nguyen-Xuan, N. Nguyen-Thanh, T.-H. Le, T. Nguyen-Thoi, and T. Rabczuk, "Static, free vibration, and buckling analysis of laminated composite Reissner-Mindlin plates using NURBS-based isogeometric approach," International Journal for Numerical Methods in Engineering, vol. 91, no. 6, pp. 571-603, 2012.

[20] H. Nguyen-Xuan, C. H. Thai, and T. Nguyen-Thoi, "Isogeometric finite element analysis of composite sandwich plates using a higher order shear deformation theory," Composites B: Engineering, vol. 55, pp. 558-574, 2013.

[21] C. H. Thai, A. J. Ferreira, E. Carrera, and H. Nguyen-Xuan, "Isogeometric analysis of laminated composite and sandwich plates using a layerwise deformation theory," Composite Structures, vol. 104, pp. 196-214, 2013.

[22] C. H. Thai, A. J. M. Ferreira, S. P. A. Bordas, T. Rabczuk, and H. Nguyen-Xuan, "Isogeometric analysis of laminated composite and sandwich plates using a new inverse trigonometric shear deformation theory," European Journal of Mechanics: A/Solids, vol. 43, pp. 89-108, 2014.

[23] L. V. Tran, C. H. Thai, and H. Nguyen-Xuan, "An isogeometric finite element formulation for thermal buckling analysis of functionally graded plates," Finite Elements in Analysis and Design, vol. 73, pp. 65-76, 2013.

[24] C. H. Thai, T. Rabczuk, and H. Nguyen-Xuan, "A rotationfree isogeometric analysis for composite sandwich thin plates," International Journal of Composite Materials, vol. 3, no. 6A, pp. 10-18, 2013.

[25] C. V. Verhoosel, M. A. Scott, T. J. R. Hughes, and R. de Borst, "An isogeometric analysis approach to gradient damage models," International Journal for Numerical Methods in Engineering, vol. 86, no. 1, pp. 115-134, 2011.

[26] P. Fischer, M. Klassen, J. Mergheim, P. Steinmann, and R. Müller, "Isogeometric analysis of 2D gradient elasticity," Computational Mechanics, vol. 47, no. 3, pp. 325-334, 2011.

[27] W. A. Wall, M. A. Frenzel, and C. Cyron, "Isogeometric structural shape optimization," Computer Methods in Applied Mechanics and Engineering, vol. 197, no. 33-40, pp. 2976-2988, 2008.

[28] P. N. Nielsen, A. R. Gersborg, J. Gravesen, and N. L. Pedersen, "Discretizations in isogeometric analysis of Navier-Stokes flow," Computer Methods in Applied Mechanics and Engineering, vol. 200, no. 45-46, pp. 3242-3253, 2011. 
[29] T. Temizer, P. Wriggers, and T. J. R. Hughes, "Contact treatment in isogeometric analysis with NURBS," Computer Methods in Applied Mechanics and Engineering, vol. 200, no. 9-12, pp. 11001112, 2011.

[30] E. D. Luycker, D. J. Benson, T. Belytschko, Y. Bazilevs, and M. C. Hsu, "X-FEM in isogeometric analysis for linear fracture mechanics," International Journal for Numerical Methods in Engineering, vol. 87, no. 6, pp. 541-565, 2011.

[31] S. S. Ghorashi, N. Valizadeh, and S. Mohammadi, "Extended isogeometric analysis for simulation of stationary and propagating cracks," International Journal for Numerical Methods in Engineering, vol. 89, no. 9, pp. 1069-1101, 2012.

[32] D. J. Benson, Y. Bazilevs, E. de Luycker et al., "A generalized finite element formulation for arbitrary basis functions: from isogeometric analysis to XFEM," International Journal for Numerical Methods in Engineering, vol. 83, no. 6, pp. 765-785, 2010.

[33] G. Haasemann, M. Kästner, S. Prüger, and V. Ulbricht, "Development of a quadratic finite element formulation based on the XFEM and NURBS," International Journal for Numerical Methods in Engineering, vol. 86, no. 4-5, pp. 598-617, 2011.

[34] S. S. Ghorashi, N. Valizadeh, and S. Mohammadi, "Analysis of cracked orthotropic media using the extended isogeometric analysis (XIGA)," in Proceedings of the 2nd European Conference on Extended Finite Element (XFEM '11), Cardiff University, Cardiff, United Kingdom, June 2011.

[35] S.-M. Hu, Y.-F. Li, T. Ju, and X. Zhu, "Modifying the shape of NURBS surfaces with geometric constraints," Computer-Aided Design, vol. 33, no. 12, pp. 903-912, 2001.

[36] A. Asadpoure, S. Mohammadi, and A. Vafai, "Crack analysis in orthotropic media using the extended finite element method," Thin-Walled Structures, vol. 44, no. 9, pp. 1031-1038, 2006.

[37] P. Liu, T. Q. Bui, Ch. Zhang, T. T. Yu, G. R. Liu, and M. V. Golub, "The singular edge-based smoothed finite element method for stationary dynamic crack problems in 2D elastic solids," Computer Methods in Applied Mechanics and Engineering, vol. 233-236, pp. 68-80, 2012.

[38] T. Menouillard and T. Belytschko, "Dynamic fracture with meshfree enriched XFEM," Acta Mechanica, vol. 213, no. 1-2, pp. 53-69, 2010.

[39] T. Elguedj, A. Gravouil, and H. Maigre, "An explicit dynamics extended finite element method. Part 1: mass lumping for arbitrary enrichment functions," Computer Methods in Applied Mechanics and Engineering, vol. 198, no. 30-32, pp. 2297-2317, 2009.

[40] T. Menouillard, S.-H. Song, Q. Duan, and T. Belytschko, “Time dependent crack tip enrichment for dynamic crack propagation," International Journal of Fracture, vol. 162, no. 1-2, pp. 3349, 2010.

[41] F. García-Sánchez, C. Zhang, and A. Sáez, "A two-dimensional time-domain boundary element method for dynamic crack problems in anisotropic solids," Engineering Fracture Mechanics, vol. 75, no. 6, pp. 1412-1430, 2008.

[42] D. Motamedi and S. Mohammadi, "Dynamic analysis of fixed cracks in composites by the extended finite element method," Engineering Fracture Mechanics, vol. 77, no. 17, pp. 3373-3393, 2010.

[43] N. Nguyen-Thanh, H. Nguyen-Xuan, S. Bordas, and T. Rabczuk, "Isogeometric analysis using polynomial splines over hierarchical T-meshes for two-dimensional elastic solids," Computer Methods in Applied Mechanics and Engineering, vol. 200, no. 2122, pp. 1892-1908, 2011.
[44] L. Chen, G. R. Liu, Y. Jiang, K. Y. Zeng, and J. Zhang, "A singular edge-based smoothed finite element method (ESFEM) for crack analyses in anisotropic media," Engineering Fracture Mechanics, vol. 78, no. 1, pp. 85-109, 2011. 


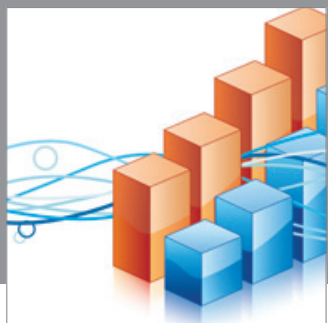

Advances in

Operations Research

mansans

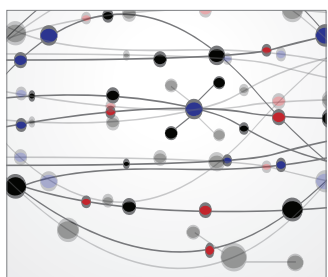

The Scientific World Journal
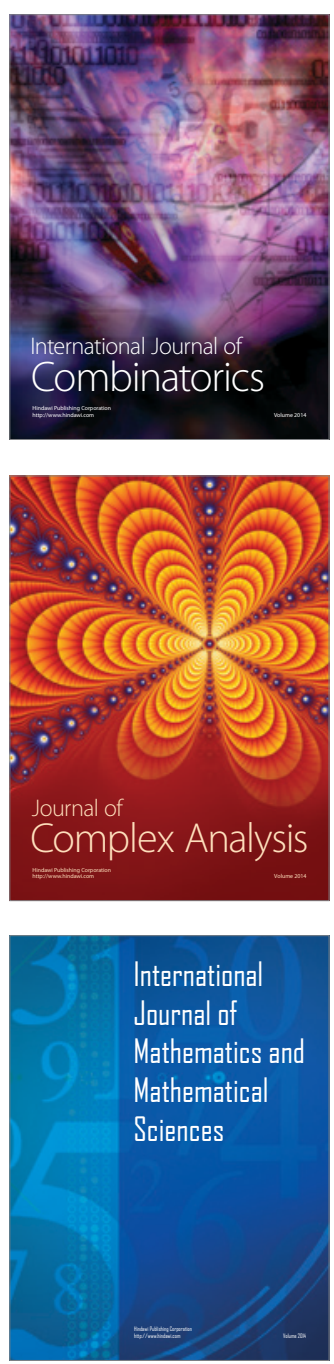
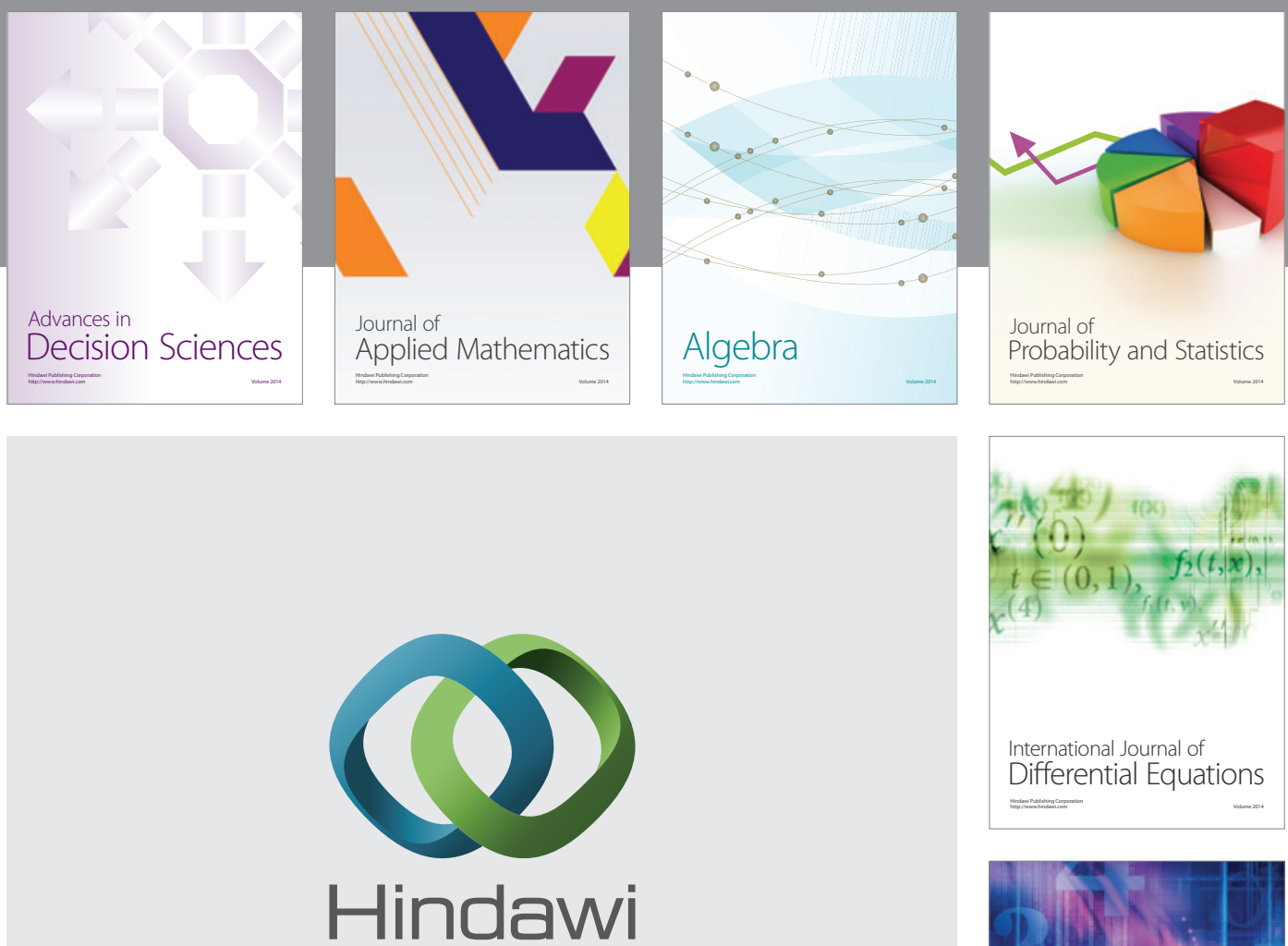

Submit your manuscripts at http://www.hindawi.com
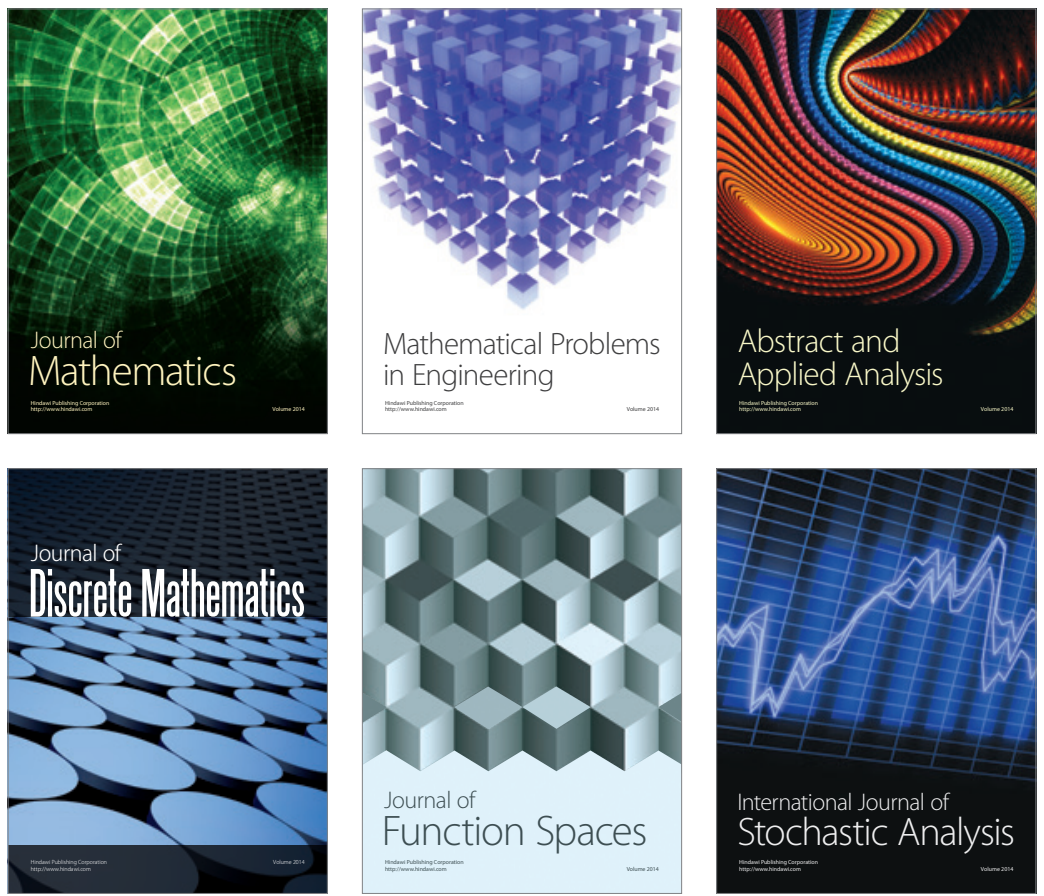

Journal of

Function Spaces

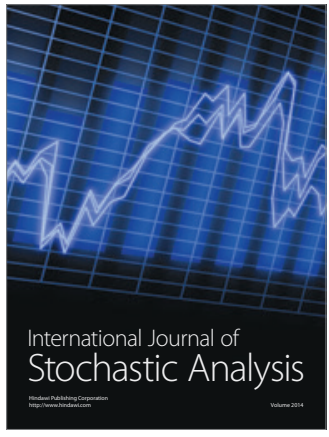

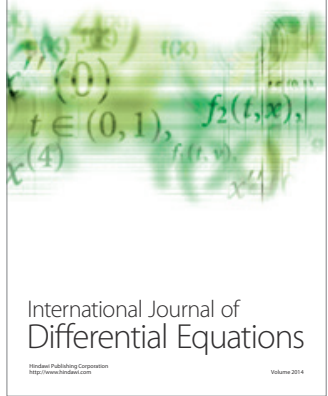
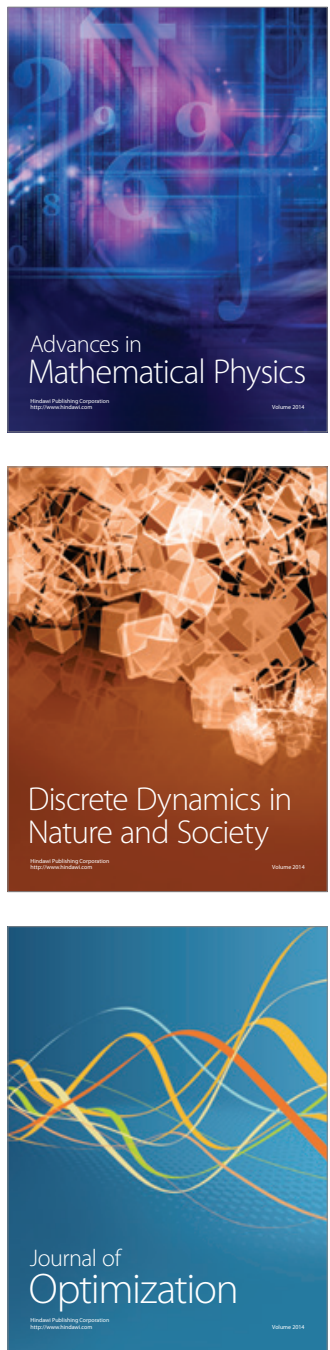